\title{
Equilibrium equation of state of a hard sphere binary mixture at very large densities using replica exchange Monte-Carlo simulations
}

\author{
Gerardo Odriozola ${ }^{1}$ and Ludovic Berthier ${ }^{2}$ \\ ${ }^{1}$ Programa de Ingeniería Molecular, Instituto Mexicano del Petróleo, \\ Lázaro Cárdenas 152, 07730 México, D. F., México \\ ${ }^{2}$ Laboratoire des Colloïdes, Verres et Nanomatériaux, \\ UMR CNRS 5587, Université Montpellier 2, 34095 Montpellier, France
}

(Dated: February 4, 2021)

\begin{abstract}
We use replica exchange Monte-Carlo simulations to measure the equilibrium equation of state of the disordered fluid state for a binary hard sphere mixture up to very large densities where standard Monte-Carlo simulations do not easily reach thermal equilibrium. For the moderate system sizes we use (up to $N=100$ ), we find no sign of a pressure discontinuity near the location of dynamic glass singularities extrapolated using either algebraic or simple exponential divergences, suggesting they do not correspond to genuine thermodynamic glass transitions. Several scenarios are proposed for the fate of the fluid state in the thermodynamic limit.
\end{abstract}

\section{INTRODUCTION}

Simple liquids, crystals, glasses, powders, and colloidal dispersions are frequently modeled using hard spheres. Although considered as one of the simplest models in condensed matter physics, hard spheres exhibit a complicated phase behavior that is not fully elucidated. In particular, a well-established first order fluid-solid transition exists in three dimensions. For monodisperse systems, it occurs from volume fractions $\varphi_{\mathrm{f}}=\pi \rho \sigma^{3} / 6 \approx 0.492$ to $\varphi_{\mathrm{s}} \approx 0.545$ ( $\rho$ is the number density and $\sigma$ the particle diameter) [1 [5]. However, the metastable fluid branch persists for $\varphi>\varphi_{\mathrm{f}}$, and its fate at large $\varphi$ remains a debated subject [6]. Since the fluid cannot exist above the maximum density of the cubic centered crystal structure, $\varphi_{\mathrm{fcc}} \approx 0.74$, it may either go unstable, or it may exhibit a singularity with a diverging (dimensionless) pressure, $Z=\beta P / \rho$, and a vanishing (also dimensionless) isothermal compressibility, $\chi=\delta \rho / \delta(\beta P)$. Here $\beta=\left(k_{B} T\right)^{-1}$, with $T$ the temperature and $k_{B}$ the Boltzmann constant. Additionally, a thermodynamic glass transition could possibly occur along the way [ 6 -11], characterized by a diverging timescale for structural relaxation [12 15], a change of slope in the equilibrium equation of state $Z(\varphi)$, and a jump in the compressibility. These features would be the analog, for hard spheres, of the glass transition observed in glassforming liquids, characterized in particular by a diverging viscosity and a jump in the specific heat [16]. It is the aim of this work to search for a thermodynamic signature of the glass transition in hard spheres.

Studying the metastable fluid branch by simulations is complicated since the system naturally tends to form the crystal phase 17, 18], at least in three dimensions [19]. Since pressure is very dependent on the existence of small amounts of crystal nuclei, excluding ordered configurations from the sampling is critical to obtain the real pressure-density relationship [17, 18]. An efficient way to overcome the somewhat arbitrary exclusion of crystalline states from the sampling is to introduce size poly- dispersity to avoid, or at least considerably delay, crystal formation. One must then work between several constraints: polydispersity must be large enough to prevent ordering, but small enough that a qualitatively different physics, specific to very polydisperse systems, does not set in. For instance, phase separation can occur in mixtures 20], or fractionation in systems with continuous polydispersity [21]. These phenomena have counterparts even for disordered states, since multiple glass transitions might occur in polydisperse systems, where for instance large particles are arrested in a sea of small ones that still easily diffuse 22]. In this work we use a 50:50 binary mixture of hard spheres with a diameter ratio 1.4, large enough to efficiently prevent crystallization, but which shows no sign of multiple glass transitions.

The final problem to be overcome is also the most difficult one: approaching the glass transition at thermal equilibrium is hard in systems where the viscosity becomes large because the timescale to reach equilibrium is simultaneously diverging. On this aspect, numerical simulations could potentially outperform experimental work since it is possible, at least in principle, to imagine algorithms that have no 'physical' counterpart but still allow a proper exploration of the configuration space, and thus of the thermodynamic properties of the system [23]. Several such 'smart' algorithms exist in various context of statistical mechanics, such as umbrella sampling which makes use of biased statistical weights, replica exchange or parallel tempering where copies of the system at various thermodynamic states are run in parallel to avoid being trapped in free energy minima 24 26], or cluster and swap algorithms which implement unphysical particle moves to speed up equilibration [27, 28].

Although commonly used and very successful in many areas of condensed matter, such methods have comparatively been much less used in numerical studies of the glass transition, for several reasons. Firstly, the glass transition is mostly defined by, and studied via, dynamic properties, and so it is vital to use physical microscopic dynamics, which inevitably yields slow dynamics. There 
are nevertheless interesting thermodynamic properties to be investigated in glassforming materials, for which implementation of particle swaps 29], cluster moves [30], Wang-Landau sampling [31, 32], or parallel tempering [33 36] have all been implemented. Of course these different methods can be combined to improve further the efficiency. This has led in particular to strong claims about both the absence [30] and presence [29, 37] of thermodynamic glass transitions in various glassy fluid models (including hard spheres), but also raised debates about the real efficiency of the various numerical algorithms to study systems with slow dynamics [33 35, 38].

Here, we employ the replica exchange Monte-Carlo (REMC) method [25, 26, 39, 40], as recently adapted to systems composed of hard particles [5]. The idea is to simulate several replicas of the same system at different but close enough thermodynamic states to allow efficient exchanges between the replicas [23]. For soft interparticle potentials, the most common ensemble expansion is that performed in temperature where each replica follows a canonical ensemble simulation and the ensembles are set at different temperatures. To take advantage of the REMC algorithm for hard spheres, one needs to expand the isobaric-isothermal ensemble in pressure [41], each replica evolving at a different pressure [5]. Replicas having the larger pressures can escape from locally stable free energy minima through successive exchanges with replicas at lower pressures [42].

Using REMC, we have been able to reach thermal equilibrium for hard spheres up to very large densities where standard Monte-Carlo algorithms do not allow proper sampling of the configuration space [15, 43, 44]. We were thus able to study the thermodynamic properties of the disordered fluid branch of a binary hard sphere mixture over a broad density range which includes both the modecoupling, $\varphi_{\mathrm{mct}}$, and Vogel-Fulcher-Tamman, $\varphi_{\mathrm{vft}}>\varphi_{\mathrm{mct}}$, dynamic singularities finding no thermodynamic signature for any of them, at least for the moderate system sizes we used, up to $N=100$. While the absence of a genuine transition at $\varphi_{\text {mct }}$ can be established by standard numerical methods [15, 43, 44], it is the main new result of this work that the same phenomenon seems to occur also at $\varphi_{\mathrm{vft}}$.

The paper is organized as follows. In Sec. II we describe in more detail the model we use, and review the various 'critical' volume fractions that have been reported in previous work. In Sec. III we provide details about the REMC simulations. In Sec. IV we perform several tests to ensure that a proper sampling of configuration space has been done. In Sec. $\nabla$ we describe our equilibrium results for the thermodynamics of the system. In Sec. VI we investigate even higher densities, for which thermal equilibration could not be reached. Finally, we discuss our results in Sec. VII

\begin{tabular}{|l|l|}
\hline Definition & Volume fraction \\
\hline Onset of glassy dynamics & $\varphi_{\text {onset }} \approx 0.56$ \\
Mode-coupling theory, Eq. (1) & $\varphi_{\text {mct }}=0.592$ \\
Vogel-Fulcher-Tamman, Eq. (2) & $\varphi_{\text {vft }}=0.615$ \\
Dynamic scaling, Eq. (3) & $\varphi_{0}=0.635$ \\
Diverging pressure (lower bound) & $\varphi_{\text {low }}=0.662$ \\
\hline
\end{tabular}

TABLE I: Values of the relevant volume fractions characterizing the physical behaviour of the fluid for the binary hard sphere mixture studied in this work.

\section{CRITICAL DENSITIES IN A BINARY HARD SPHERE MIXTURE MODEL}

Previous work on binary mixtures suggests that a 50:50 binary mixture of hard spheres with a diameter ratio of 1.4 is a very efficient way to prevent crystalline ordering even at large densities [7, 44, 45]. We will use $N=$ $N_{A}+N_{B}$ particles, $N_{A}$ and $N_{B}$ denoting the number of small and large particles in the mixture, respectively. We work in units where the diameter of the small particles is unity, $\sigma_{A A}=1$.

Moreover, the dynamics of small and large particles is strongly coupled so that the slow relaxation and location of the putative dynamic glass singularities yields consistent results for both components of the mixture [15, 46]. Thus, this model seems well-suited for investigating the existence of a thermodynamic glass transition of hard spheres. In a previous (bidimensional) study where efficient cluster Monte-Carlo moves were used [30], a very large polydispersity was introduced, with the unwanted result that large particles seemed to arrest at a density where small particles could still easily diffuse, making the identification of dynamic singularities somewhat ambiguous [38].

Previous numerical explorations of the dynamics of the present binary mixture revealed the existence of very slow dynamics and possible dynamic singularities at large volume fraction, with no interference from the crystalline phase [15]. Several relevant values of the packing fractions have been reported using different definitions and theoretical approaches, and we summarize them in Table [1]

First, the dynamics of the system slows down and starts to become non-exponential above $\varphi_{\text {onset }} \approx 0.56$, which can thus be seen as the onset density for slow dynamics in this system.

Second, the location of several dynamic 'singularities' can be defined and have been numerically studied. An algebraic divergence of the relaxation time,

$$
\tau \sim\left(\varphi_{\mathrm{mct}}-\varphi\right)^{-\gamma},
$$

as predicted by mode-coupling theory [47], can be located near $\varphi_{\text {mct }} \approx 0.592$. However, simulations also revealed this density to be a crossover since the equilibrium relaxation time can be measured at and above $\varphi_{\text {mct }}$ where it remains finite [15, 46]. This suggests that a different 
functional form should be used to extrapolate a possible divergence of the relaxation time.

A popular functional form for $\tau(\varphi)$ is the so-called Vogel-Fulcher-Tamman (VFT) expression [16],

$$
\tau \sim \tau_{\infty} \exp \left(\frac{A}{\varphi_{\mathrm{vft}}-\varphi}\right),
$$

which yields, for the present system, the value $\varphi_{\mathrm{vft}} \approx$ $0.615, A$ and $\tau_{\infty}$ being additional fitting parameters [15]. As opposed to the mode-coupling singularity, standard simulations fail to access such a large packing fraction in equilibrium conditions, since the largest state point investigated in Ref. [15] is $\varphi=0.597<\varphi_{\text {vft }}$.

Using a combination of scaling arguments involving both direct simulations of hard particles, and a soft harmonic repulsion at very low temperatures, recent numerical work provided support for the existence of a slightly different, stronger dynamic divergence [15, 44, 46, 48],

$$
\tau \sim \tau_{\infty}\left(\frac{A}{\left(\varphi_{0}-\varphi\right)^{\delta}}\right)
$$

with the preferred values $\delta \approx 2.2$ and $\varphi_{0} \approx 0.635$.

Finally, taking the view that no thermodynamic glass transition occurs, one must conclude that dynamics should arrest when particles come into contact and no particle move can take place. In this perspective, $\tau$ must diverge simultaneously with the pressure $Z$ at the random close packing or jamming density, $\varphi_{\mathrm{rcp}}$, which can then be empirically defined as the end point of the equilibrium equation of state of the fluid branch [49]. In practice this is hard to measure because the system falls out of equilibrium and becomes a nonergodic hard sphere glass much before getting to jamming, such that only lower bounds to the location of the diverging pressure can be numerically determined. For the present system, previous work reported the value $\varphi_{\text {low }} \approx 0.662$ as the tightest lower bound on $\varphi_{\mathrm{rcp}}$, obtained by rapid compressions of carefully equilibrated fluid states [44, 50]. This result indicates that the putative end point of the metastable fluid branch for this system is above $\varphi_{\text {low }}=0.662$.

\section{THE REPLICA EXCHANGE MONTE-CARLO METHOD}

The partition function in the extended ensemble studied in the replica exchange Monte-Carlo method we use is given by [5, 41]

$$
Q_{\text {extended }}=\prod_{i=1}^{n_{r}} Q_{N T P_{i}},
$$

where $Q_{N T P_{i}}$ is the partition function of the isobaricisothermal ensemble of the system at pressure $P_{i}$, temperature $T$, particle number $N$. The important new parameter is $n_{r}$, the considered number of replicas of the system.
This extended ensemble is sampled by combining standard NTP $P_{i}$ simulations on each replica (involving both trial displacements of single particles and trial volume changes) and replica exchanges (swap moves at the replica level). To satisfy detailed balance, these swap moves are performed by setting equal all a priory probabilities for choosing adjacent pairs of replicas and using the following acceptance probability [5, 41]

$$
P_{\text {acc }}=\min \left(1, \exp \left[\beta\left(P_{i}-P_{j}\right)\left(V_{i}-V_{j}\right)\right]\right),
$$

where $V_{i}-V_{j}$ is the volume difference between replicas $i$ and $j$. Adjacent pressures should be close enough to provide nonnegligible exchange acceptance rates between neighboring ensembles. In order to take good advantage of the method, the ensemble at the smaller pressure must also ensure large jumps in configuration space, so that the larger pressure ensembles can be efficiently sampled.

The probability for selecting a particle displacement trial, $P_{d}$, for selecting a volume change trial, $P_{v}$, and a swap trial, $P_{s}$, are fixed to

$$
\begin{aligned}
& P_{d}=n_{r} N /\left(n_{r}(N+1)+w\right), \\
& P_{v}=n_{r} /\left(n_{r}(N+1)+w\right), \\
& P_{s}=w /\left(n_{r}(N+1)+w\right),
\end{aligned}
$$

where $w \ll 1$ is a weight factor. Note that $P_{d}+P_{v}+P_{s}=$ 1 , as it should. The probability density function to have the next swap trial move at the trial $n_{t}$ is given by

$$
P\left(n_{t}\right)=P_{s} \exp \left(-P_{s} n_{t}\right) .
$$

Hence, one may obtain the next swap trial move from $n_{t}=-\ln (\xi) / P_{s}$, with $\xi$ being a random number uniformly distributed in the interval ]0,1] [51, 52]. We set all particles of a given replica to have the same a priori probability of being selected to perform a displacement trial. The same is true for selecting a replica for performing a volume change trial.

The trials $\left[1, n_{t}-1\right]$ are displacements and volume changes, and so, they can be independently performed on the replicas. This has the advantage of being easily parallelized. The algorithm is parallelized in four threads, since quad core desktops are used, but could be more efficiently parallelized in $n_{r}$ threads. Since all swap trials are performed in a single core, the efficiency of the parallelization increases with decreasing $w$. We employed $w=1 / 100$. Verlet lists are used for saving CPU time, which can be quite large for the replicas evolving with the highest pressure values.

Our simulations are performed in two steps. All simulations are started by randomly placing particles (avoiding overlaps), so that the initial volume fraction is $\varphi=$ 0.30 . We first perform about $2 \times 10^{13}$ trial moves at the desired state points, during which we observe that the replicas reach a stationary state. We then perform more $2 \times 10^{13}$ additional trials during which various measurements are performed, with results described in the following sections. 

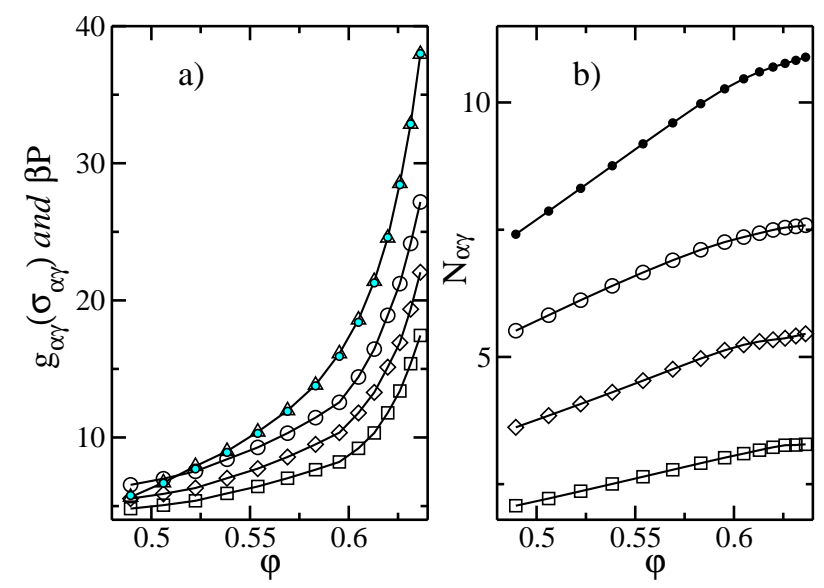

FIG. 1: a) $g_{A A}\left(\sigma_{A A}\right)$ (squares), $g_{A B}\left(\sigma_{A B}\right)$ (diamonds), and $g_{B B}\left(\sigma_{B B}\right)$ (circles) as a function of $\varphi$. The total pressure, Eq. (8), is shown as triangles. It agrees well with the set pressure values (light bullets). b) The number of AA (squares), $\mathrm{AB}$ (diamonds), and $\mathrm{BB}$ (circles) neighbors as a function of $\varphi$ with bullets indicating the total number of neighbors per particle. All data correspond to $N=100$.

The maximum particle displacements and volume changes for trial moves are adapted for each pressure to yield acceptance rates close to 0.3. Thus, particle displacements and volume changes of ensembles having high pressures are smaller than those associated to ensembles having low pressures. An optimal allocation of the replicas should lead to a constant swap acceptance rate for all pairs of adjacent ensembles. For a temperature expansion, the efficiency of the method peaks at swap acceptance rates close to $20 \%$ [53]. In this work, we use instead a geometric progression of the pressure with the replica index. In Sec. $\mathrm{V}$ we report results for various system sizes, $N=60$, 80, and 100 using $n_{r}=14$, with $\beta P$ varying from 38 to approximately 5.8 , the geometrical factor being 0.865 . In Sec. VI we present additional results where the largest pressure is $\beta P=100, N=60$, $n_{r}=18$, and the geometrical factor is 0.840 .

\section{THERMALIZATION TESTS}

The aim of this work is to provide new, reliable thermodynamic information at large densities where thermalization becomes a severe issue for standard algorithms. This means in particular that the algorithm must be able to sample accurately a phase space where ergodicity is potentially broken in the thermodynamic limit. Such severe sampling conditions are also met for instance in systems such as spin glasses [40]. It is crucial to establish whether the produced results are indeed representative of thermal equilibrium, as we now discuss.

As a first check we verify that the pressure measured from the configurations sampled by the replicas in each ensemble yield results consistent with the values set nu-

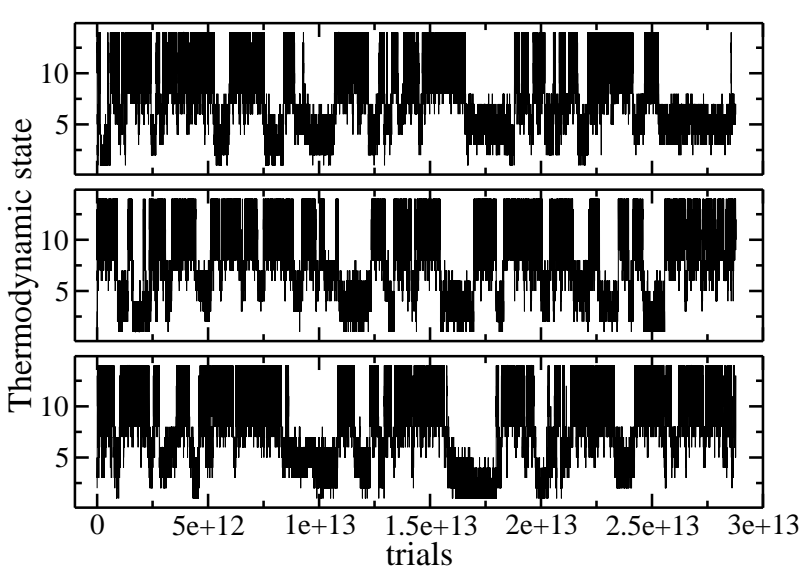

FIG. 2: Random walk in pressure space for $N=100$ and $n_{r}=14$ for three arbitrarily chosen replicas. Thermodynamic state ' 1 ' corresponds to the highest pressure and ' 14 ' to the lowest. All replica visit several times both lowest and highest pressure states.

merically. Pressure and structure are related by 20]

$$
\frac{\beta P}{\rho}=1+\frac{2 \pi \rho}{3} \sum_{\alpha} \sum_{\gamma} x_{\alpha} x_{\gamma} \sigma_{\alpha \gamma}^{3} g_{\alpha \gamma}\left(\sigma_{\alpha \gamma}\right)
$$

where $\alpha$ and $\gamma$ run over species $A$ and $B$, and $x_{\alpha}, \sigma_{\alpha \gamma}$, and $g_{\alpha \gamma}$ respectively being the fraction of particles in species $\alpha$, the contact distance between $\alpha$ and $\gamma$, and the partial radial distribution functions of species $\alpha$ and $\gamma$. Note that $g_{\alpha \gamma}\left(\sigma_{\alpha \gamma}\right)$ must be evaluated using a careful extrapolation of $g_{\alpha \gamma}(r)$ towards contact. Thus, we may split the excess pressure into three contributions, corresponding to the $\mathrm{AA}, \mathrm{AB}$, and $\mathrm{BB}$ interactions. These contributions are shown in Fig. 1fa together with the total pressure obtained from Eq. (8). As can be seen, the measured pressure agrees very well with the values imposed numerically. Furthermore, a smooth behavior is obtained for all $g_{\alpha \gamma}\left(\sigma_{\alpha \gamma}\right)$ as a function of $\varphi$ suggesting that adequate sampling has been performed.

For all $\varphi$ the largest contribution to the excess pressure is that of the large-large pairs (BB), followed by the large-small $(\mathrm{AB})$ and the small-small (AA) pairs, in that order. In the right panel of Fig. 1 the evolution of the average number of neighbors is shown, obtained by integration of the partial pair correlation functions in a spherical shell of constant thickness $0.2 \sigma_{A A}$. The total number of neighbors per particle is also shown. The numbers of neighbors are consistent with the contributions to the excess pressure, i. e., they increase following the order $\mathrm{AA}, \mathrm{AB}$, and $\mathrm{BB}$ at all $\varphi$ and they increasing with $\varphi$, albeit more slowly than the pressure. This saturation is physically expected since the number of neighbors remains finite even when the pressure diverges near jamming.

We mentioned in the introduction that preventing crystallization is in principle dealt with by using a binary mixture. Since evidence for this stems from standard numerical approaches, it remains to be seen whether REMC 


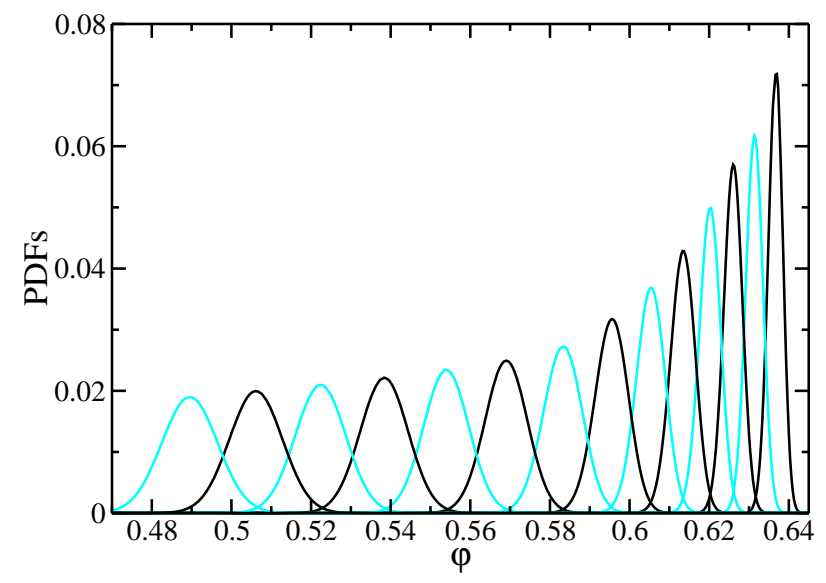

FIG. 3: Probability distribution functions (PDFs) of volume fraction fluctuations for each of the $n_{r}=14$ pressure values, $N=100$. All distributions are stationary, featureless, and symmetric and have sufficient overlap to allow for replica exchanges.

finds more easily the crystalline phase or not. Indeed previous work on the monodisperse system showed that the REMC algorithm is not only capable of forming the crystal phase but also to accurately predict the liquid-solid transition [5]. Thus, if a crystal is the preferred state, we expect to see signs of local orientational order. We checked this by computing the well-known order parameter $Q_{6}$, as defined for instance in Refs. [5, 18, 54], which is very sensitive to any trace of local angular order [18]. We evaluate it separately for $\mathrm{AA}, \mathrm{BB}$, and $\mathrm{AB}$ pairs. In all cases and at all densities, $Q_{6}$ is very close to the value of a completely random system of points [18]. Moreover, the three $Q_{6}$ values do not evolve significantly during the runs. Thus, we can safely conclude that if the crystal phase corresponds to the equilibrium state of this particular binary mixture, it is sufficiently metastable not to affect our results regarding the disordered state.

For replica exchange methods to provide an efficient sampling of phase space, it is important to check whether all simulated replicas visit the entire set of thermodynamic conditions several times. This is a necessary condition for thermalization because this ensures that the configurations contributing to the thermodynamic averages are very different as the low pressure replicas evolve rapidly and have large displacements in configuration space. In Fig. 2 we show the evolution of three randomly selected replicas making a random walk among the different pressure states. We observe that all replicas contribute several times during the course of the production run to both the highest and the lowest pressure states. With the imposed geometric progression of the pressure, we find that the acceptance rate has a small drop near $\varphi \approx 0.58$, which suggests that thermalization becomes much harder above these densities. It is intriguing that this corresponds roughly to $\varphi_{\mathrm{mct}}$, above which it also becomes hard to reach thermal equilibrium using standard
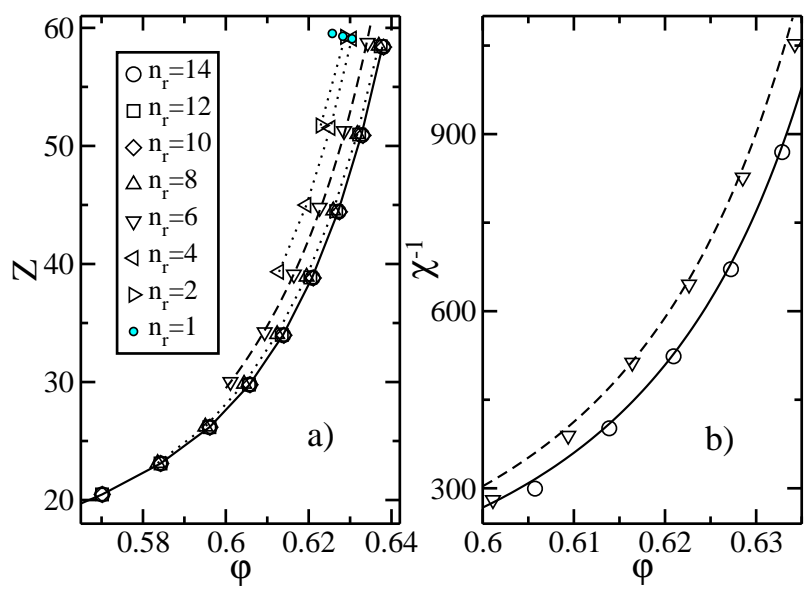

FIG. 4: a) Equation of state, $Z=\beta P / \rho=Z(\varphi)$, for $N=60$ and increasing number of replicas from $n_{r}=1$ to $n_{r}=14$. Only data for $n_{r}>8$ are reproducible, while data for a smaller $n_{r}$ are not thermalized. b) Checking the FDT relation, Eq. (9), for density fluctuations. The lines show $1 / \chi$ obtained from taking the derivative of the equation of state, while symbols are direct evaluation using spontaneous fluctuations of the density. The FDT holds with good accuracy both for equilibrated systems $\left(n_{r}=14\right.$, bottom $)$ and for nearly frozen ones $\left(n_{r}=6\right.$, top).

numerical tools. This numerical bottleneck suggests a faster decrease of the number of accessible configurations as pressure increases, or a faster increase of the barriers separating long-lived metastable states.

Each replica evolves at a given predefined pressure $P_{i}$. Therefore, the volume $V_{i}$ of replica $i$ is a fluctuating quantity, and it is interesting to focus on the probability density functions (PDFs) of the volumes $V_{i}$, or equivalently of the corresponding volume fractions $\varphi_{i}$. In cases where the system remains trapped in long-lived metastable states, the PDFs may be distorted or may contain peaks or shoulders which help detecting a lack of thermalization. Additionally, these features of the PDFs typically disappear as time increases and thus help revealing whether measurements are performed in stationary states. We observe that the PDFs evolve in the first simulation steps but they then become both symmetric and take a Gaussian shape. The resulting functions are shown in Fig. 3 for $N=100$ with low volume fractions PDFs correspond to low pressures. Notice that the PDFs have a larger peak and become narrower as pressure increases, which reflects the fact that the compressibility decreases.

Ergodicity implies that the same results should be obtained independently of the set initial conditions and of the parameters of the simulation. We checked the reproducibility of our results by running simulations with $n_{r}=1,2,4,6,8,10,12$, and 14 , for $N=60$, for the same highest pressure $(\beta P=38)$ and geometrical factor of 0.865 . Three independent runs were carried out with $n_{r}=1$, having all different initial conditions. For a fair comparison, all simulations lasted four weeks running 
on identical single cores and no parallelization was implemented for this particular test. From the measured PDFs at each pressure, we measure the averaged density to calculate the equation of state $Z(\varphi)$, which are reported in Fig. 4. We observe that results become reproducible only when $n_{r} \geq 8$, which corresponds to simulations where the lowest pressure is below $\varphi=0.58$ and yields thermalized results. For smaller $n_{r}, Z$ is always larger than that obtained for $n_{r} \geq 8$, suggesting that thermal equilibrium had not been reached. In particular, the three independent runs with $n_{r}=1$ are well above the equilibrated curve and distinct from one another. This implies that runs with $n_{r}<8$ are nonergodic and do not sample the configuration space accurately at large densities even with a large number of trials. In particular, this means that a standard Monte-Carlo algorithm would not yield equilibrium results at large density, and that it is clearly outperformed by the REMC simulation scheme we use in this work.

A final test for equilibrium was suggested by Santen and Krauth [30]. At thermal equilibrium the spontaneous fluctuations of density are related to the isothermal compressibility, which is defined from the response of the pressure to an infinitesimal change in density in the linear response regime. This relation is thus a form of fluctuation-dissipation theorem (FDT), which is derived using the hypothesis that states are sampled with the equilibrium Gibbs measure:

$$
\chi=N\left(\frac{\left\langle\rho^{2}\right\rangle-\langle\rho\rangle^{2}}{\langle\rho\rangle^{2}}\right)=\frac{\delta \rho}{\delta(\beta P)} .
$$

Checking whether this relation is satisfied by the data is therefore in principle a good way to check thermalisation. In practice, this means checking the existence of a quantitative relationship between the broadness of the PDFs in Fig. 3 and the location of their averages.

We followed these two routes for obtaining $\chi$. The fluctuations are directly measured from the simulations, while the response function is obtained by first fitting the pressure locally to a smooth polynomial function before taking the derivative with respect to density. In Fig. 4 we present our results showing $1 / \chi$ as a function of density using both methods. When $n_{r}=14$ and results are reproducible, we find that the FDT relative to fluctuation density is well satisfied, which comes as an additional proof that our data are representative of thermal equilibrium. However we note that for runs with a small number of replica all concentrated in the high density regime which appeared far from equilibrium in the left panel of Fig. 4 the FDT is also satisfied with a good accuracy. In that case, all replicas belong to the glassy state and are basically frozen in a single 'basin' where they sample quasi-equilibrium short-lived fluctuations. Studies of FDT violations in aging glasses have indeed shown that deviations from FDT appear only when considering those degrees of freedom that relax very slowly in the glass 55]. This suggests that the FDT test suggested in Ref. [30] is only effective in a narrow density regime where a complete separation of timescale does not make Eq. (9) valid even very far from equilibrium. That is, the test seems useful to detect a slow evolution and thus, the consistency of both $\chi$ determinations only guarantees a stationary state has been reached. This is a necessary but insufficient condition for equilibrium.

\section{THERMODYNAMIC RESULTS AT EQUILIBRIUM}

In previous sections, we provided evidence that the REMC algorithm is properly implemented, and that it might give thermalized results for $N=100, n_{r}=14$ up the pressure $\beta P=38$. In this section we study more carefully the outcome of this study, starting with the equation of state $Z(\varphi)$.

Using the PDFs shown in Fig. 3 it is easy to deduce the average volume fraction for each pressure, and thus to obtain $Z(\varphi)$. The results are shown in Fig. 5-a for three different systems sizes $N=60,80$ and 100. A comparison of the three system sizes shows that finite size effects appear to be very small for the equation of state as the data obtained with different system sizes practically coincide. Nevertheless, larger system sizes produce a very small but apparently systematic decrease of the volume fraction at a given pressure, for all $\varphi$. As a further check, we report the results of an independent study using a standard Monte-Carlo approach which used $N=1000$, but covers a smaller range of pressures [44]. Up to $\varphi \approx$ 0.595 where both data sets can be compared, the data agree very well, confirming the validity of our algorithm, at least up to this density.

A first quantitative result from our study stems from data at volume fractions larger than the ones studied in Ref. [4], which were all accurately described using the Boublik-Mansoori-Carnahan-Starling-Leland (BMCSL) equation of state [56, 57], which is the extension for mixtures of the Carnahan-Starling equation of state. The data in Fig. 5ta follow the BMCSL equation up to $\varphi \approx 0.59$, but clearly deviate from it at larger volume fractions, the deviations becoming very large at large $\varphi$ where BMCSL clearly underestimates the pressure. A similar deviation was recently reported from molecular dynamics simulations of a hard sphere system with continuous polydispersity [58].

As a better description of the data at large $\varphi$ we used the fitting formula suggested from free volume considerations [59],

$$
Z=\frac{d^{\prime} \varphi_{c}}{\varphi_{c}-\varphi}
$$

where $d^{\prime}$ and $\varphi_{c}$ are free fitting parameters. Although the prefactor $d^{\prime}$ should in principle be constrained within free volume theory to be equal to the spatial dimension $d$, we find that its value must be adjusted to describe our data. In Fig. 5 - a we show the best fit to the data $\varphi>0.61$ to Eq. (10) as a full line, using $d^{\prime}=2.82$ and 

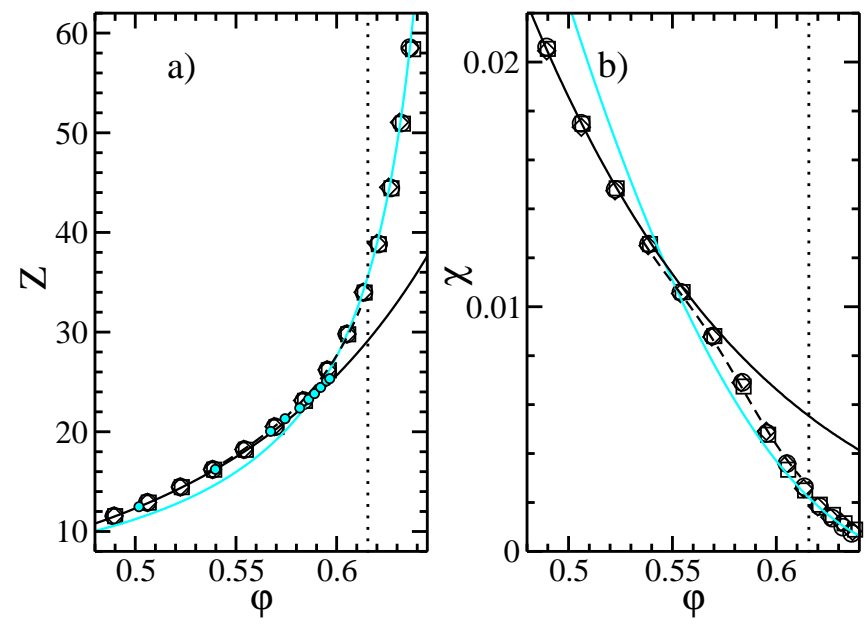

FIG. 5: a- Equation of state $Z(\varphi)$ for different system sizes, $N=60$ (open squares), $N=80$ (circles), and $N=100$ (diamonds). Light bullets are $N=1000$ data taken from Ref. 44]. The black solid line is the BMCSL equation of state, the dashed line an empirical polynomial form, and the light line a simple pole divergence, Eq. (10). b- Isothermal compressibility obtained from density fluctuations (symbols) or by derivative of the fits shown in panel a. The vertical dotted line is at $\varphi_{\mathrm{vft}}$, where no thermodynamic signature of a glass transition is found.

$\varphi_{c}=0.669$. This value of $\varphi_{c}$ should be compared to the lower bound for the diverging pressure discussed above in Sec. II (see Table I) which was $\varphi_{\text {low }}=0.662$ [50]. The large difference between the two values is a direct sign that the REMC algorithm has been able to thermalize the system much more efficiently. Note also that the fit in Eq. (10) only works at large volume fractions, while at low $\varphi$ values it clearly deviates from the simulation data.

Finally to account for the crossover region $\varphi \approx 0.58-$ 0.61 between the BMCSL and free volume fits, we use an empirical high order polynomial fit, shown with a dashed line. We give no particular emphasis on a physical interpretation of this fit, which we simply use as a fitting tool to obtain the numerical derivative of the pressure, and thus the compressibility, in this intermediate Range of volume fractions.

A second important result of our study is obtained by considering the vertical line which corresponds to the volume fraction $\varphi_{\mathrm{vft}}=0.615$. While an extrapolation of the relaxation time divergence using Eq. (2) indicates the possibility of a glass transition occurring at $\varphi_{\mathrm{vft}}$, there is no corresponding thermodynamic signature in the equation of state, in particular no sign that a kink develops as the system size increases, at least for the modest $N$ values we have been able to study, see Sec. VII.

These results are confirmed in Fig. 5-b which shows the evolution of the isothermal compressibility as a function of $\varphi$ for the different system sizes. These data are directly obtained from the spontaneous density fluctuations, i. e., from $\chi=N\left(\left\langle\rho^{2}\right\rangle-\langle\rho\rangle^{2}\right) /\langle\rho\rangle^{2}$, and they directly confirm the absence of any jump in the compressibility over this range of volume fractions and system sizes, in particular near $\varphi_{\text {vft }}$.

We also show in this figure the compressibility values as obtained from $\delta \rho / \delta(Z \rho)$, using the three fits described above, namely using the BMCSL equation of state at low $\varphi$ (black line), the polynomial fit at intermediate $\varphi$ (dashed line) and the free volume fit at high $\varphi$ (light line). There is excellent agreement between both sets of data showing that Eq. (9) is well satified at over the entire range of volume fractions.

The compressibility data simply amplify the results obtained for $Z(\varphi)$. In particular, the good agreement between the BMCSL equation of state and the numerical data is very good up to $\varphi \approx 0.56$, but deviations in fact already appear at moderate volume fractions $\varphi \approx 0.57-0.59$, that are not obvious from the pressure itself (see Fig. 57a). Similarly, the free volume description of the data is only adequate above $\varphi=0.61$. These two limits make evident the existence of a crossover regime $\varphi \sim 0.56-0.61$ where neither approaches work, and the only description we have is an empirical polynomial function, which, interestingly, shows two changes of the concavity but no jump.

To sum up, our simulation data at low and intermediate densities agree with the BMCSL equation of state, while at large densities they are much better described by a simple divergence at $\varphi_{c}=0.669$. The data show no jump of $\chi$ in the studied range of $\varphi$ values, which encompasses both $\varphi_{\text {mct }}$ and $\varphi_{\text {vft }}$ fitted dynamic singularities.

\section{INCREASING THE PRESSURE FURTHER: NONEQUILIBRIUM EFFECTS}

In the previous section we found the unexpected result that, for modest system sizes, equilibrium data could be produced even beyond the fitted location of the VFT singularity. In this section we ask whether it is possible to go to even larger volume fractions and cross $\varphi_{0}=$ 0.635 , the putative location of the thermodynamic glass transition estimated in Ref. [44].

To start answering this question we run a simulation with $N=60$ and a larger number of replicas, $n_{r}=18$ setting the maximum pressure to $\beta P=100$ and a geometric factor of 0.84 . As before, we discard the first $2 \times 10^{13}$ trials, and use $2 \times 10^{13}$ trial moves to perform measurements.

The results for $Z(\varphi)$ and $\chi(\varphi)$ are shown in Fig. 6. while the pressure, contact values of the radial distribution functions, and number of neighbors are shown in Fig. 7. The data shown in Fig. 6] are consistent with those found previously with $n_{r}=14$. There is not only a good agreement with the data obtained for $n_{r}=14$ and $N=100$, but also with the free volume extrapolation towards larger $\varphi$. Additionally, for $\varphi<0.63$, the measured pressure shown in Fig. [7 a matches the imposed pressure and all structural quantities display a smooth evolution with $\varphi$, see Fig. 7fb. Unfortunately, this smooth behavior 

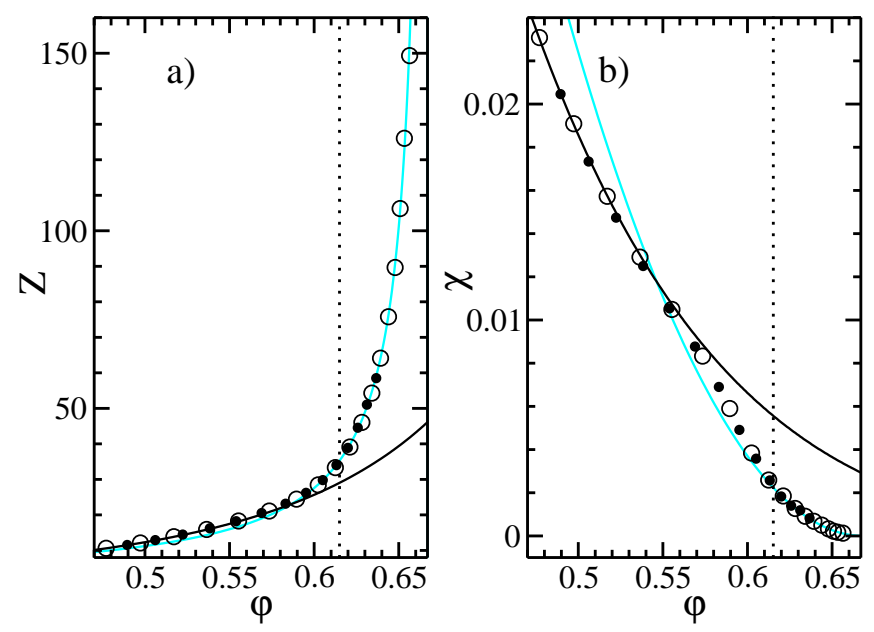

FIG. 6: Same as Fig. 5 comparing runs with $\left(N=100, n_{r}=\right.$ 14 , maximum pressure $\beta P=38$, filled circles $)$ to $(N=60$, $n_{r}=18$, maximum pressure $\beta P=100$, open circles). Black lines correspond to the BMCSL equation of state, light lines to free volume fit Eq. (10), the vertical line denotes $\varphi_{\mathrm{vft}}=0.615$. While both data sets coincide below $\varphi \approx 0.63$, the $n_{r}=18$ data at large pressures have not reached thermal equilibrium.
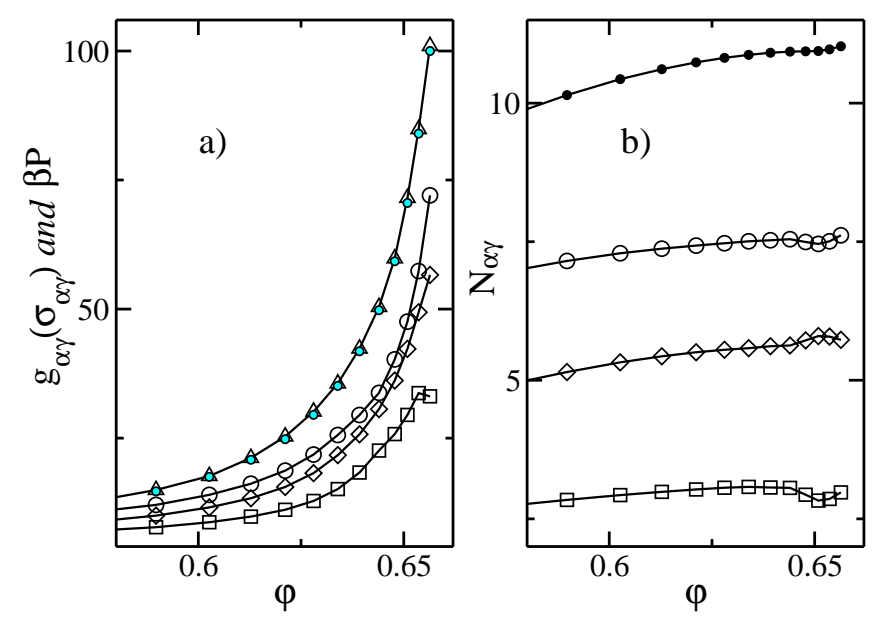

FIG. 7: Same as Fig. 1 for the run with $N=60$ and $n_{r}=18$. The data scatter at large volume fraction, $\varphi>0.63$ indicates nonergodic effects.

is lost for $\varphi \gtrsim 0.63$, see Fig. [7] suggesting that inadequate sampling is performed.

This conclusion is further supported by the data shown in Fig. 8 which shows the path in the pressure space of three chosen replicas. Despite an acceptance rate for replica exchanges being close to $10 \%$, the replicas clearly do not sample all thermodynamic states with equal probability. In particular, it is clear that the averages at large pressure are performed over a very limited number of independent configurations, suggesting that an ergodic sampling of the phase space is not achieved. Note that the third replica, before getting arrested at large pressure near the end of the run, smoothly travels among the

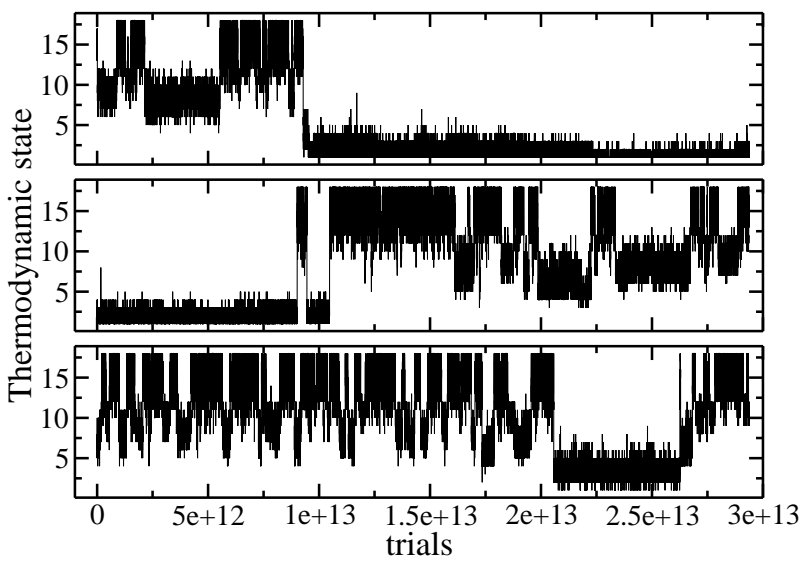

FIG. 8: Same as Fig. 2 for the run with $N=60$ and $n_{r}=18$. Thermodynamic state ' 1 ' corresponds to the highest pressure and ' 18 ' to the lowest. Contrary to Fig. 2 here the replica do not appropriately visit all thermodynamic states in an ergodic manner.

highest 14 pressures, which probably explains why the two data sets with $n_{r}=14$ and $n_{r}=18$ produce consistent results below $\varphi=0.63$, despite the fact that the latter run is clearly not producing fully thermalized data.

Therefore, we conclude that much longer simulations would be needed to reach thermal equilibrium above $\varphi \approx 0.63$, presumably with a larger number of replicas to allow a more extensive sampling of the configuration space. Unfortunately, this implies that despite our numerical effort we cannot discuss the possibility raised in Ref. [44] that a thermodynamic glass transition takes place near $\varphi_{0}=0.635$ in the present binary hard sphere mixture.

\section{DISCUSSION}

In this work, we have demonstrated that the replica exchange Monte-Carlo method recently adapted to improve the sampling of hard sphere systems is a useful new tool to investigate the thermodynamic behaviour of the disordered fluid state in a binary mixture of hard spheres up to very large volume fractions. We found that reproducible, thermalized results could be obtained up to $\varphi \approx 0.63$ at least for moderate system sizes, $N \leq 100$. This volume fraction is beyond two important 'critical' packing fractions defined dynamically, namely the mode-coupling transition $\varphi_{\mathrm{mct}}=0.592$ and the divergence extrapolated using a Vogel-Fulcher-Tamman expression, $\varphi_{\mathrm{vft}}=0.615$. Following the equation of state for the pressure $Z(\varphi)$ and the isothermal compressibility $\chi(\varphi)$ we found no signature of a thermodynamic glass transition up to $\varphi=0.63$ for the system sizes we use. Additionally, we have pushed the lower bound for the location of the divergence of the pressure of the fluid branch up to $\varphi=0.669$, much above the previous determination $\varphi=0.662$.

For computational reasons our study was limited both 
in the range of system sizes and of volume fractions for which thermal equilibrium could be reached. Thus, our results leave open the existence of (at least) three different scenarios for the behaviour of the fluid of hard spheres at large volume fractions in the thermodynamic limit.

In a first scenario, we assume that finite size effects are small and that our data at large pressure above $\varphi=0.63$ are nevertheless indicative that no change of behaviour is to be expected for $Z$ and $\chi$ even at larger volume fractions, such that Eq. (10) will continue to hold up to some $\varphi_{c}$. In this view, $\varphi_{c}$ would represent the end point of the fluid branch where the equilibrium pressure of the fluid would diverge, while the region $\varphi=0.56-0.61$ represents a crossover from the BMCSL equation of state to a free volume-like divergence. To prove or disprove this scenario is nearly impossible, as one should establish that no thermodynamic singularity occurs up to $\varphi_{c}$ in the thermodynamic limit. It is also natural to expect, in this perspective, that the equilibrium relaxation time of the fluid would also diverge at $\varphi_{c}$. This was termed the "jamming" scenario in Ref. [44] because it is the diverging pressure that controls the divergence of the viscosity. Note that our results imply that this divergence will in any case occur above $\varphi=0.669$, which is much above the location of the jamming transition ('point J') at $\varphi_{J}=0.648$ obtained using purely athermal packing preparation protocols [60]. Thus, even in the absence of a thermodynamic glass transition, point $\mathrm{J}$ does not control the glass transition of hard spheres.

A second scenario could be that finite size effects are severe, that our checks with different system sizes are insufficient, and that $N=100$ is still very far away from the thermodynamic limit even in the crossover regime $\varphi=0.58-0.62$. In that case, the crossover region we have described could potentially become sharper, in the ther- modynamic limit, yielding a discontinuity of the pressure and a jump of the compressibility. This scenario is potentially simpler to study numerically based on our work, as one should attempt to increase further the range of system sizes studied while maintaining thermal equilibrium in the crossover regime, an objective that does appear numerically realistic.

In a third scenario, our conclusions would continue to hold in the thermodynamic limit up to $\varphi=0.63$, confirming in particular that nothing special happens near $\varphi_{\mathrm{vft}}=0.615$. However, a thermodynamic transition could still take place at larger density, as suggested for instance in Refs. [15, 44, 46, 48] where a dynamic singularity was located near $\varphi_{0}=0.635$. Although we found no thermodynamic signature of $\varphi_{0}$ in Sec. VI we also noticed that our data at these large volume fraction were not thermalized leaving open the possibility that a pressure discontinuity exists at equilibrium. Exploring this third scenario would be quite demanding, as one would need to cross $\varphi_{0}$ at thermal equilibrium for larger systems.

To conclude, it should come as no surprise that providing solid conclusions regarding the existence of a thermodynamic liquid-glass transition in the thermodynamic limit is a difficult numerical task. However, we have provided evidence that replica exchange Monte-Carlo simulations can be used to study this issue in hard sphere systems, and we have suggested that drawing some firm conclusions is perhaps not completely out of reach. In particular, it would be interesting to study larger system sizes, together with a larger number of replicas to maintain the acceptance rates for replica exchanges at an acceptable level. This implies using larger computational resources.
[1] B. J. Alder and T. E. Wainwright, J. Chem. Phys. 27, 1208 (1957).

[2] W. G. Hoover and F. H. Ree, J. Chem. Phys. 49, 3609 (1968).

[3] N. B. Wilding and A. D. Bruce, Phys. Rev. Lett. 79, 3002 (1997).

[4] E. G. Noya, C. Vega, and E. de Miguel, J. Chem. Phys. 128, 154507 (2008).

[5] G. Odriozola, J. Chem. Phys. 131, 144107 (2009).

[6] G. Parisi and F. Zamponi, Rev. Mod. Phys. 82, 789845 (2010).

[7] R. J. Speedy, J. Chem. Phys. 100, 6684 (1994).

[8] J. Yeo, Phys. Rev. E. 52, 853 (1995).

[9] A. van Blaaderen and P. Wiltzius, Science 270, 1177 (1995).

[10] R. J. Speedy, J. Phys.: Condens. Matter 9, 8591 (1997).

[11] L. Angelani and G. Foffi, J. Phys.: Condens. Matter 19, 256207 (2007).

[12] P. N. Pusey and W. van Megen, Phys. Rev. Lett. 59, 2083 (1987).

[13] W. van Megen, T. C. Mortensen, S. R. Williams, and J.
Müller, Phys. Rev. E 58, 6073 (1998).

[14] Z. Cheng et al., Phys. Rev. E 65, 041405 (2002).

[15] G. Brambilla et al., Phys. Rev. Lett. 102, 085703 (2009).

[16] P. G. Debenedetti and F. H. Stillinger, Nature 410, 259 (2001).

[17] M. D. Rintoul and S. Torquato, Phys. Rev. Lett. 77, 4198 (1996).

[18] M. D. Rintoul and S. Torquato, J. Chem. Phys. 105, 9258 (1996).

[19] J. A. van Meel, B. Charbonneau, A. Fortini, and P. Charbonneau, Phys. Rev. E 80, 061110 (2009).

[20] T. Biben and J. P. Hansen, Phys. Rev. Lett. 66, 2215 (1991).

[21] P. Sollich and N. B. Wilding, Phys. Rev. Lett. 104, 118302 (2010).

[22] A. Imhof and J. K. G. Donth, Phys. Rev. Lett. 75, 1662 (1995).

[23] D. Frenkel and B. Smit, Understanding molecular simulation (Academic, New York, 1996).

[24] Y. Sugita and Y. Okamoto, Chem. Phys. Lett. 329, 261 (2000). 
[25] K. Hukushima and K. Nemoto, J. Phys. Soc. Jpn. 65, 1604 (1996).

[26] R. H. Swendsen and J.-S. Wang, Phys. Rev. Lett. 57, 2607 (1986).

[27] W. Krauth, Algorithms and computations (Oxford University Press, Oxford, 2006).

[28] E. P. Bernard, W. Krauth, and D. B. Wilson, Phys. Rev. E 80, 056704 (2009).

[29] T. S. Grigera and G. Parisi, Phys. Rev. E 63, 045102 (2001).

[30] L. Santen and W. Krauth, Nature 405, 450 (2000).

[31] J. Ghosh and R. Faller, J. Chem. Phys. 128, 124509 (2008).

[32] R. Faller and J. J. de Pablo, J. Chem. Phys. 119, 4405 (2003).

[33] R. Yamamoto and W. Kob, Phys. Rev. E 61, 5473 (2000).

[34] K. K. Bhattacharya and J. P. Sethna, Phys. Rev. E 57, 2553 (1998).

[35] C. De Michele and F. Sciortino, Phys. Rev. E 65, 051202 (2002).

[36] E. Flenner and G. Szamel, Phys. Rev. E 73, 061505 (2006).

[37] Q. Yan, T. S. Jain, and J. J. de Pablo, Phys. Rev. Lett. 92, 235701 (2004).

[38] Y. Brumer and D. R. Reichman, J. Phys. Chem. B 108, $6832(2004)$.

[39] A. P. Lyubartsev, A. A. Martsinovski, S. V. Shevkunov, and P. N. Vorontsov-Velyaminov, J. Chem. Phys. 96, 1776 (1992).

[40] E. Marinari and G. Parisi, Europhys. Lett. 19, 451 (1992).

[41] T. Okabe, M. Kawata, Y. Okamoto, and M. Mikami, Chem. Phys. Lett. 335, 435 (2001).

[42] Q. L. Yan and J. J. de Pablo, J. Chem. Phys. 111, 9509
(1992)

[43] E. Flenner and G. Szamel, arXiv:1005.3794 (2010).

[44] L. Berthier and T. A. Witten, Phys. Rev. E. 80, 021502 (2009).

[45] D. N. Perera and P. Harrowell, Phys. Rev. E 59, 5721 (1999).

[46] D. El Masri et al., J. Stat. Mech. P07015 (2009).

[47] W. Götze, Complex dynamics of glass-forming liquids: A mode-coupling theory (Oxford University Press, Oxford, 2008).

[48] L. Berthier and T. A. Witten, Europhys. Lett. 86, 10001 (2009).

[49] R. D. Kamien and A. J. Liu, Phys. Rev. Lett. 99, 155501 (2007).

[50] P. Chaudhuri, L. Berthier, and S. Sastry, Phys. Rev. Lett. 104, 165701 (2010).

[51] D. T. Gillespie, J. Chem. Phys. 81, 2340 (1994).

[52] G. Odriozola et al., J. Phys. Chem. B 107, 2180 (2003).

[53] N. Rathore, M. Chopra, and J. J. de Pablo, J. Chem. Phys. 122, 024111 (2005).

[54] P. J. Seinhardt, D. R. Nelson, and M. Ronchetti, Phys. Rev. B. 28, 784 (1996).

[55] L. F. Cugliandolo, J. Kurchan, and L. Peliti, Phys. Rev. E 55, 3898 (1997).

[56] T. Boublik, J. Chem. Phys. 53, 471 (1970).

[57] G. A. Mansoori, N. F. Carnahan, K. E. Starling, and T. W. Leland, J. Chem. Phys. 54, 1523 (1971).

[58] M. Hermes and M. Dijkstra, J. Phys.: Condens. Matter 22, 104114 (2010).

[59] W. Salsburg and W. Wood, J. Chem. Phys. 37, 798 (1962).

[60] C. S. O'Hern, S. A. Langer, A. J. Liu, and S. R. Nagel, Phys. Rev. Lett. 88, 075507 (2002). 